\title{
Experiment on the thermocapillary convection of a mercury liquid bridge in a floating half zone
}

\author{
J.H. Han ${ }^{\mathrm{a}, *}$, Z.W. Sun ${ }^{\mathrm{b}}$, L.R. Dai ${ }^{\mathrm{c}}$, J.C. Xie ${ }^{\mathrm{a}}$, W.R. Hu ${ }^{\mathrm{a}}$ \\ ${ }^{a}$ Institute of Mechanics, Chinese Academy of Sciences, Beijing 100080, People's Republic of China \\ ${ }^{\mathrm{b}}$ General Establishment of Space Science and Applications, Chinese Academy of Sciences, Beijing 100080, People's Republic of China \\ ' Department of Chemistry, Peking University, Beijing 100871, People's Republic of China
}

Received 11 July 1995; accepted 23 January 1996

\begin{abstract}
A liquid bridge of a floating half zone consisting of liquid mercury sealed in a glass tube with nitrogen atmosphere was used for the experiment of thermocapillary convection with a low Prandtl number liquid. A non-contacted diagnostic method was developed to monitor the surface flow and the surface oscillation. A growing surface film (or skin) is a crucial source to suppress thermocapillary convection, and is discussed in this paper. For the case of a mercury liquid bridge, the critical Marangoni number was obtained as 900 , and the oscillatory frequency was around $5 \mathrm{~Hz}$.
\end{abstract}

\section{Introduction}

Marangoni convection is driven by the non-uniformity of the surface tension due to a temperature or concentration gradient along the free surface of a liquid. The liquid bridge of a floating half zone is a typical model to simulate the surface tension driven flow of crystal growth processes performed in a floating zone configuration. The steady and oscillatory convection of molten materials during materials processing are considered as one of the main causes, which may produce crystal defects and inhomogeneities. The convection driven by buoyancy is greatly reduced in a microgravity environment, and the convection driven by the surface tension gradient becomes dominant. The ground-based experiment

\footnotetext{
${ }^{*}$ Corresponding author
}

with a small typical scale is usually used to emphasize the thermocapillary effect in comparison with the buoyant effect and then to test the processes in the microgravity environment.

A great number of publications have been devoted to thermocapillary convection and its instability in the past decades. However, most experimental works on this area are limited to transparent liquids with a Prandtl number around 1 or larger, see for example Refs. [1-5]. On the other hand, unfortunately, melts of semiconductors, metals or alloys are related to the behavior of liquids having low Prandtl numbers, where the Prandtl number Pr is defined as

$\operatorname{Pr}=\nu / \kappa$,

and $\nu$ and $\kappa$ are respectively the viscosity and the thermal diffusion coefficient. Difficulties in experiments of liquids with $\operatorname{Pr}<1$ include flow visualization due to non-transparency and sensitivity of their surface to impurities. Only a few experiments of 
thermocapillary convection with low Pr can be found in the literature [6-13]. Jurisch used an electron beam heating facility to study the surface temperature oscillations of oscillatory thermocapillary convection in a floating Mo full zone $(\mathrm{Pr}=0.025)$ and found that the critical Marangoni number for the onset of oscillations is 1200 and the frequency is around $1 \mathrm{~Hz}[10,11]$. Major experimental results on the onset of oscillation for a floating full zone are summarized in Table 1. Nakamura et al. estimated that, in their experiments of a silicon floating zone, the typical oscillation frequency is $0.5 \mathrm{~Hz}$ [13], however, the experimental Marangoni number was much higher than the critical Marangoni number.

The experimental set-up of floating half zone convection was designed especially for liquid bridge experiments of low Prandtl number fluids to study the onset of oscillation. Mercury was adopted as experimental medium due to its liquid state at room temperature and its chemical stability. The special difficulty associated with the floating half zone bridge is how to choose the material of the rods which hold the liquid bridge. Ideally, chemically inert rods, such as graphite or stainless steel, are preferred to avoid a reaction with mercury. However, the wettability of the rod material to mercury is essential to form the bridge that means both materials should have a certain affinity. We found no way to use those inert rod materials because they lack the necessary affinity with mercury. Finally copper was chosen for its relatively low solubility in mercury and affinity with mercury. However, it is impossible to prevent mercury from the formation of amalgam. To minimize the effect of the surface film, we have chosen lower experimental temperatures and a shorter experimental duration.
Optical methods, that were used to measure the critical state of the onset of oscillation for silicon oil experiments of floating half zone convection $[4,5]$, could be modified for the use in the experiment of a non-transparent medium as given in the present experiment. The Marangoni number is defined as

$\mathrm{Ma}=\left|\sigma_{T}^{\prime}\right| \Delta T l / \rho \kappa \nu$,

where $\sigma$ and $\rho$ are respectively the surface tension and the density of the liquid, $l$ and $\Delta T$ are respectively the height and applied temperature difference of the liquid bridge, and $\sigma_{T}^{\prime}=\partial \sigma / \partial T$. Generally, the implication of transition from the steady flow into an oscillatory one in the low Pr case is complicated in comparison with the process for larger Pr number liquids. An interesting phenomenon, that is, the liquid metal covered with a surface film (skin), was studied in the present experiment. The possibility of a skin suppressing surface flow was also discussed on another occasion by Schwabe et al. [14].

In this paper, a brief description of the experimental arrangement will be discussed in the next section, and some experimental results are given in Section 3. The last section includes discussion and conclusion.

\section{Experimental procedure}

The liquid bridge consisting of highly purified mercury was adopted as the experimental medium, and the physical properties of mercury are listed in Table 2 . The mercury was held by surface tension between two co-axial cylindrical copper rods of $d_{0}$ $=3 \mathrm{~mm}$ in diameter. The height $l$ of the liquid bridge can be adjusted in the system and therefore the aspect ratio $A=l / d_{0}$. Another geometrical pa-

Table 1

Results of floating full zone experiments

\begin{tabular}{|c|c|c|c|c|c|c|}
\hline Material & $\operatorname{Pr}$ & $\begin{array}{l}\text { Height } \\
(\mathrm{mm})\end{array}$ & $\begin{array}{l}\text { Diameter } \\
(\mathrm{mm})\end{array}$ & $(\mathrm{Ma})_{\mathrm{c}}$ & $\begin{array}{l}f_{\mathrm{c}} \\
(\mathrm{Hz})\end{array}$ & Ref. \\
\hline $\mathrm{Ti}_{3} \mathrm{Cu}$ & - & 12.0 & 6.0 & - & 0.03 & {$[8]$} \\
\hline $\mathrm{Si}$ & 0.02 & 14.0 & 10.0 & $100-200$ & $\sim 1$ & [9] \\
\hline \multirow[t]{3}{*}{ Mo } & 0.025 & 3.4 & 2.6 & 638 & $\sim 1$ & {$[10]$} \\
\hline & 0.025 & 4.1 & 4.0 & 925 & $\sim 1$ & {$[10]$} \\
\hline & 0.025 & 5.1 & 6.0 & 1625 & $\sim 1$ & [10] \\
\hline $\mathrm{Nb}$ & 0.025 & 4.0 & 6.0 & 1025 & $\sim 1$ & [11] \\
\hline GaAs & 0.068 & 7.6 & 6.0 & $400-500$ & $\sim 0.3$ & [12] \\
\hline
\end{tabular}


Table 2

Thermo-physical parameters of mercury at $20^{\circ} \mathrm{C}$

\begin{tabular}{ll}
\hline Density $\rho$ & $1.355 \times 10^{4} \mathrm{~kg} / \mathrm{m}^{3}$ \\
Surface tension $\sigma$ & $0.4761 \mathrm{~N} / \mathrm{m}$ \\
Heat capacity $C_{p}$ & $139.08 \mathrm{~J} / \mathrm{kg} \cdot \mathrm{K}$ \\
Thermal conductivity $k$ & $8.33 \mathrm{~J} / \mathrm{m} \cdot \mathrm{s} \cdot \mathrm{K}$ \\
Thermal diffusivity $\kappa$ & $4.42 \times 10^{-6} \mathrm{~m}^{2} / \mathrm{s}$ \\
Dynamical viscosity $\nu$ & $1.14 \times 10^{-7} \mathrm{~m}^{2} / \mathrm{s}$ \\
Prandtl number Pr & 0.0258 \\
Expansion coefficient $\beta$ & $0.182 \times 10^{-3} \mathrm{~K} /$ \\
$\partial \sigma / \partial T$ & $0.19 \times 10^{-3} \mathrm{~N} / \mathrm{m} \cdot \mathrm{K}$ \\
\hline
\end{tabular}

rameter (see, for example, Ref. [15]), the volume of the liquid bridge $V$, may also be selected and $V / V_{0}$ is adopted as a critical parameter in the present paper, where $V_{0}$ is the volume of a exactly cylindrical shape with the same aspect ratio $A$.

The system of the floating half zone was sealed in a glass tube filled with highly pure $\mathrm{N}_{2}$ (impurities in it are less than $0.001 \%$ ). The end surface of the copper rods was treated with nitric acid to get a clean surface, then the surface was rubbed against a mercury droplet. This procedure improves the wettability of the copper surface with mercury. The upper rod is heated by a resistance heater and the lower rod is cooled with ice water during the experiment. Temperatures at the end of these two rods are measured by a copper-constantan thermocouple, however, there was no thermocouple inserted into the liquid bridge of mercury. (See Fig. 1, inside of the copper rod there is a hole conducting the thermocouples to the end wall of the rod.) According to the depth of the end wall $(d)$, the thermal conductivity of copper and mercury, and the size of the mercury bridge, we can calculate the temperature at the interface between the copper rod and the mercury. The system of the mercury floating half zone is schematically shown in Fig. 1.

Non-contacted optical methods were developed for non-transparent mediums. To detect the deformation and oscillation of the free surface, a laser beam is aimed at the surface of the mercury liquid bridge. The reflected light from the curved surface is projected on a screen. The resultant enlarged interference images on the screen are monitored by a CCD camera and recorded by a video recorder in real time. Then, the variation of the interference patterns associated with the configurations of the free surface will change accordingly. In this way, even a very

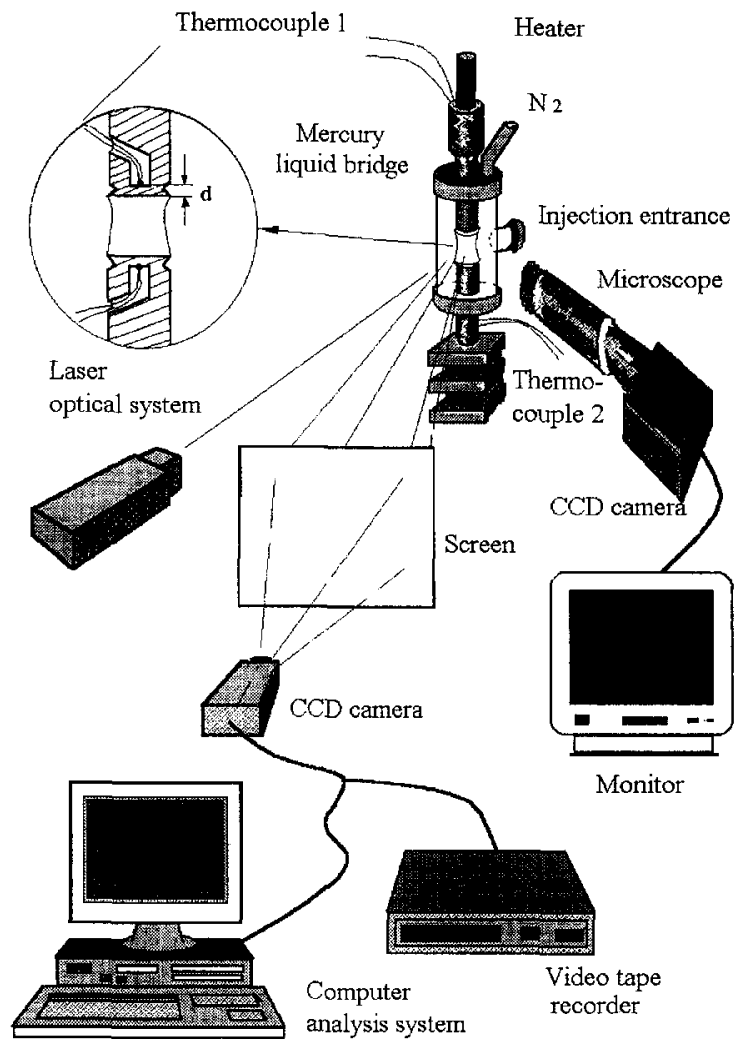

Fig. 1. Schematic diagram of the experimental system.

small deformation of the free surface can be specified in real time. A typical sample of a so obtained interference pattern at one moment is shown in Fig. 2.

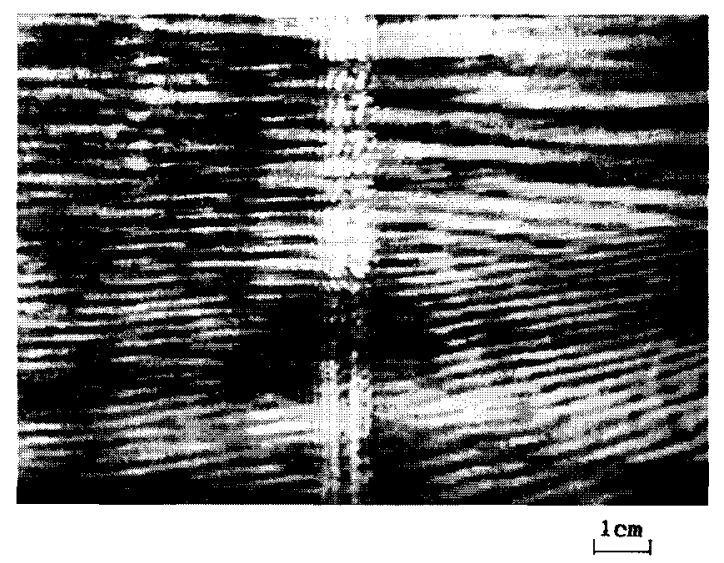

Fig. 2. Interference pattern associated with the free surface configuration. 
In order to obtain the frequency of the oscillatory convection, a special photocell was developed. The interference pattern varies after the onset of oscillation. In the present experiment, the oscillatory variation of the interference pattern was recorded on a video tape, and the photocell was aimed at one of the interference fringes and fixed in place to detect the variations in the brightness resulting in a variation of the output of the photocell when the fringe was moving back and forth. The oscillation of the mercury liquid bridge was obtained successfully by the photocell.

It seems to be a necessary step to check whether there is a skin growing on the mercury surface. Dusts, impurities or even pieces of growing skin absorbed on the mercury surface can be observed by the present optical method, although the free surface looked quite bright and smooth with the naked eye. The onset of oscillation will obviously be constrained by the skin, and may be observed when the skin is broken. Generally, two critical Marangoni numbers $(\mathrm{Ma})_{c}^{+}$and $(\mathrm{Ma})_{c}^{-}$can be defined respectively by the transition process from the steady to the oscillatory state associated with increasing applied temperature difference and the one from oscillatory to steady state associated with decreasing applied temperature difference [16]. The values of these two critical Marangoni numbers are different in general, but are close for the transparent medium experiments. It seems that the Marangoni number $(\mathrm{Ma})_{c}^{-}$ would make more sense for the liquid with a wrapped skin. The properties and influence of the skin will be discussed in detail elsewhere.

Dusts or impurities may play the role of tracers to display the surface flow. With the aid of illumination of a $\mathrm{He}-\mathrm{Ne}$ laser, movements of these "tracers" can clearly be seen by a microscopic facility. The surface flow pattern depends jointly on the thermocapillary convection, the temperature profile at the surface and the structure and composition of the skin. The high surface energy of mercury enriches impurities on the surface, resulting in a surface film which obstructs the surface flow. The impurities include coppermercury amalgam and, probably, oxidic mercury because oxygen can not be absolutely excluded in this system. This skin growing process can be observed by the transition of the tracers from moving to rest.

\section{Results}

After clean mercury (without any pollutant or skin on the surface) is injected into the gap between the two rods, the liquid bridge is formed. When a temperature difference is imposed between the two rods, the surface flow can be seen by watching the movements of the "tracers". With an increase of the applied temperature difference, the tracer flow speeds up at the beginning and then slows down, and eventually the mobility of the surface vanishes within a period ranging from a couple of minutes to ten minutes depending on the applied temperature. This phenomenon seems to be related to the formation of a thin film (or skin) on the surface. A similar phenomenon that a "dirt-film" suppressed the surface flow was also observed experimentally by Schwabe et al. [14]. Their experimental medium was n-tetracosane $\mathrm{C}_{24} \mathrm{H}_{50}$ with $\mathrm{Pr}=49$ and they found that the free surface was covered with a dirt-film and the fluid was motionless for all the $\Delta T^{\prime}$ below $\Delta T=60$ $\mathrm{K}$. Only after the $\Delta T$ reached $60 \mathrm{~K}$ the dirt-film ruptured and the surface flow became possible.

After several experiments we put all used mercury together and carefully separated the surface film from the bulk mercury. An atomic emission spectroscope (Jarrell-Ash III Type, Fisher Scientific Company, US) was used to semi-quantitatively determine the impurity contents of the film. The result showed that this film has about several thousandths of copper (in addition to its main composition mercury) that implies that copper-mercury amalgam may be the main impurity. When the temperature of the mercury liquid bridge is high, the film is formed quickly because the higher temperature will accelerate the reaction of mercury with the copper rods.

The oscillation associated with a certain applied temperature difference and without surface mobility was observed in the mercury liquid bridge of the floating half zone. Experiments were performed for different typical parameters. A typical evolution of the oscillation with variation of applied temperature difference for the case of $A=l / d_{0}=0.6$ and $V / V_{0}$ $=0.814$ is shown in Fig. 3, where the solid and the broken line are associated respectively with the oscillatory and steady states and the square mark means the moment of onset or offset of oscillation. It can be seen that there is a relative high value of $(\mathrm{Ma})_{c}^{+}$at 


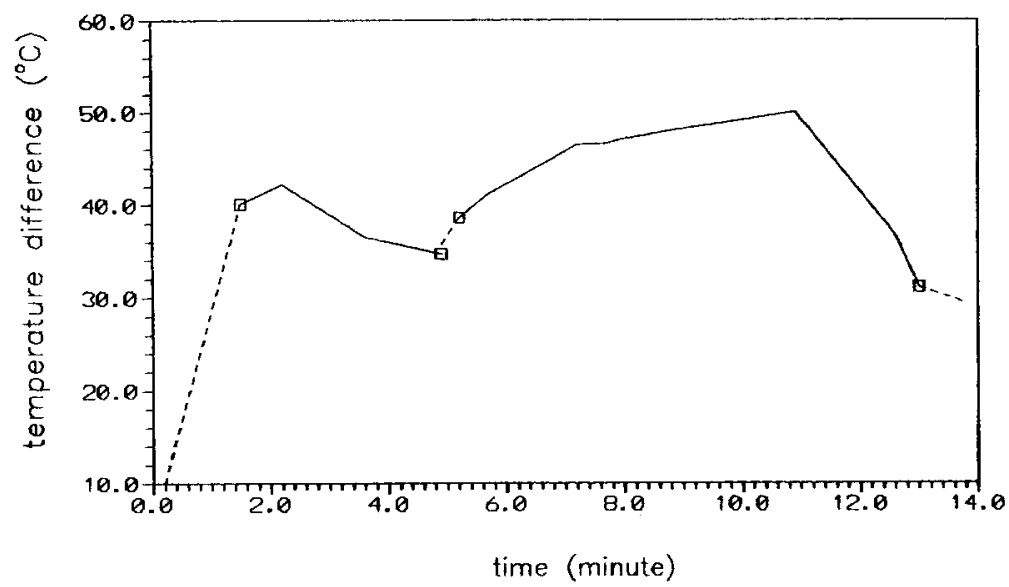

Fig. 3. Process associated with onset and offset of oscillation during the evolution of the applied temperature difference. (The broken line represents a steady state and the solid line corresponds to an oscillatory state.)

the beginning. The reason of this high value comes probably from the influence of the skin, which tends to obstruct the onset of oscillation. After the skin is broken, the oscillation keeps on until a much lower applied temperature difference $(\Delta T)_{\mathrm{c}}^{-}$or $(\mathrm{Ma})_{\mathrm{c}}^{-}$. The $(\mathrm{Ma})_{\mathrm{c}}^{-}$corresponding to the above mentioned sample is about 1700 . Even after the skin was broken, the feature of the surface, such as $\partial \sigma / \partial T$, could possibly be affected by other pollutants because of the high sensitivity of the surface. All of these contribute uncertainties to the experiment. Several experiments were performed with a little different geometric parameters, and gave different critical Marangoni numbers. The existence of the skin would tend to increase the $(\Delta T)_{c}$, and thus the lowest $(\mathrm{Ma})_{\mathrm{c}}^{-}$would be a more reasonable estimation because of minimum skin disturbance. In the present experiment it gives

$(\mathrm{Ma})_{\mathrm{c}}^{-} \approx 900$.

There are theoretical suggestions on the critical Marangoni number for the case of a cylindrical liquid bridge, $V / V_{0}=1$, in a microgravity environment. The conditions of ground-based experiments cannot be satisfied to the standard of theoretical models, however, the comparison may be worthwhile. The results of the present paper agree in order of magnitude with that of the numerical simulation for a silicon floating zone [17], but are different in order of magnitude from the linear analyses of instability [18].

The oscillation process of the surface is shown in Fig. 4, obtained by the photocell measurement of the brightness variation of the interference fringes, which gave voltage outputs varying with time. Fig. 5 is the frequency spectrum analysis of Fig. 4, which shows a main frequency of

$f_{\mathrm{m}}=5 \mathrm{~Hz}$.

However, from the oscillation process shown in Figs. 4 and 5 we can see that the frequency is not a constant, but spreads in the range of 5 to $10 \mathrm{~Hz}$. The frequency obtained in this experiment is higher than that given in Refs. [8-11].

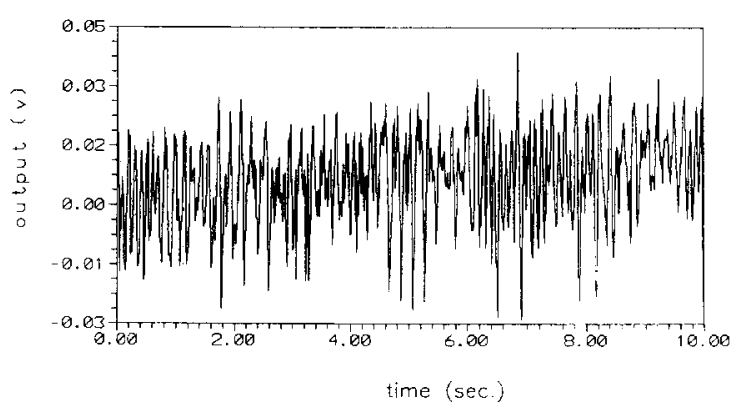

Fig. 4. Oscillatory profile of the photocell output associated with free surface oscillation for a longer period. 


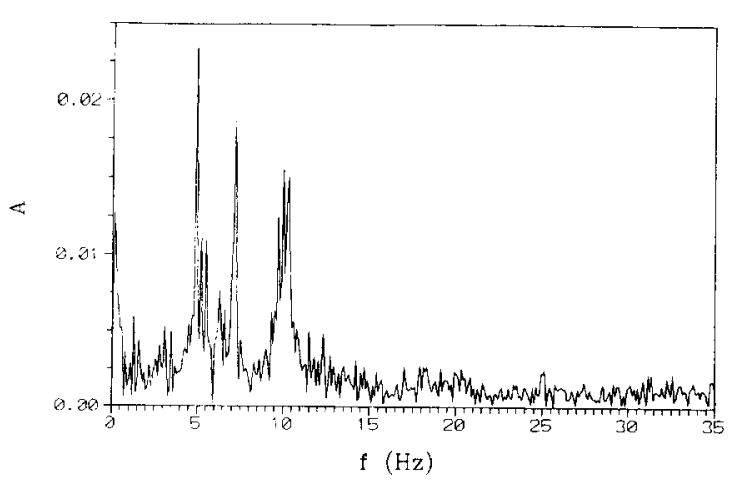

Fig. 5. Frequency spectrum of the oscillation associated with Fig. 4.

An estimation of the main frequency was given by dimension analyses as [4]

$f_{\mathrm{c}}=(\nu+\kappa) / l^{2}$

and it gives $f_{\mathrm{c}}=1.4 \mathrm{~Hz}$ which agrees in order of magnitude with the experimental result (3.2).

\section{Discussion and conclusion}

Thermocapillary convection in a mercury liquid bridge of a floating half zone is studied experimentally. In the literature, only a few experiments of liquid bridges with small Prandtl number fluids are described and they are all limited to the full floating zone arrangement. Probably the present paper is the first one to discuss experimentally the thermocapillary convection and its oscillatory feature for the model of a floating half zone formed by a low Prandtl number liquid.

In the present work non-contacted measurements were used successfully to monitor the free surface variation. The oscillatory frequency in the present experiment is one order of magnitude higher than the one in Ref. [13], where the frequency was measured by an inserted thermocouple and the experimental Marangoni number was much higher than the critical Marangoni number. It was pointed out that an inserted thermocouple will influence the heat transfer and therefore the oscillatory feature in the silicon oil liquid bridge of a half floating zone [4], and it could be expected that the influence will be more serious in the liquid bridge of metals such as mercury.
Therefore, the non-contacted diagnostic method is more favorable in the experiment of thermocapillary convection, especially with small Prandtl number liquids.

The surface film often appears in the floating zone experiments for smaller Prandtl number liquids, and the critical Marangoni number will be much larger for the case with a global film in comparison with the case of a broken film. It seems that the critical Marangoni number given by the offset of oscillation is rather reasonable to describe the transition state. It looks like the oscillations came around the locations where the skin was broken during the onset process of oscillation, and the periodicity of this oscillations seems not to be a perfect sine type probably because of the influence of pieces of the skin. In comparison with high Prandtl number liquids, the frequency of the oscillation is higher due to the larger value of the thermal diffusion coefficient. It seems that more attention should be paid to the analyses of surface concentration and impurities in future research. As mentioned above, this kind of experiment involves special difficulties mainly connected to formation of a surface film which causes uncertain factors in the experimental process. A reasonable inference of the present experiment is that the uncertainties related to the skin or pollution to the surface of the liquid bridge would be a general problem for thermocapillary convection of low $\mathrm{Pr}$ liquids.

\section{References}

[1] C.E. Chang, W.R. Wilcox, J. Crystal Growth 28 (1975) 8.

[2] C.-H. Chun, W. Wuest, Acta Astronautica 5 (1978) 681.

[3] R. Velten, D. Schwabe, A. Scharmann, Proc. 7th Eur. Symp. on Materials and Fluid Science in Microgravity, ESA SP-295, 1990, p. 271.

[4] Z.H. Cao, J.C. Xie, Z.M. Tang, W.R. Hu, Adv. Space Res. 11 (1991) 161.

[5] Z.H. Cao, H.T. You, Z.M. Tang, W.R. Hu, Adv. Space Res. 11 (1991) 232.

[6] J. Barthel, K. Eichlen, J. Crystal Growth 64 (1974) 565.

[7] D.T.J. Hurle and E. Jakeman, J. Crystal Growth 5 (1969) 227.

[8] Y.K. Chang, J Crystal Growth 62 (1983) 627.

[9] A. Cröll, W. Müller-Sebert, R. Nitsche, Proc. 7th Eur. Symp. on Materials and Fluid Science in Microgravity, ESA SP-295, 1990, p. 263. 
[10] M. Jurisch, W. Löser, J. Crystal Growth 102 (1990) 214.

[11] M. Jurisch, J. Crystal Growth 102 (1990) 223.

[12] R. Rupp, S. Aurochs, G. Müller, C. Weyrich, S. Leibenzeder, Adv. Space Res. 11 (1991) 297.

[13] S. Nakamura, K. Kakimoto, T. Hibiya, Proc. 9th Eur. Symp. on Gravity Dependent Phenomena in Physical Sciences, Berlin, May 1995, p. 16.

[14] D. Schwabe, A. Scharmann, Adv. Space Res. 8 (1988) 175.

[15] W.R. Hu, J.Z. Shu, R. Zhou, Z.M. Tang, J. Crystal Growth 142 (1994) 379
[16] D. Schwabe, A. Scharmann, XXV COSPAR Topical Meeting G.1, Paper G.1.2.4. Recent Scientific Results and Developments of Materials Sciences in Space, July 1984, Graz, Austria; Z. Flugwiss, Weltraumforschung 9 (1985) 21.

[17] N.D. Kazarinoff, J.S. Wilkowski, Phys. Fluid A 2 (1990) 1797.

[18] H.C. Kuhlmann, H.J. Rath, J. Fluid Mech, 147 (1993) 247. 